Engineered acoustic mismatch for anchor loss control in contour mode resonators

A. Lozzi, A. De Pastina, E. Ting-Ta Yen and L. G. Villanueva

This article may be downloaded for personal use only. Any other use requires prior permission of the author and AIP Publishing. This article appeared in A. Lozzi et al., Appl. Phys. Lett. 114, 103502 (2019) and may be found at https://doi.org/10.1063/1.5086156 


\section{Engineered acoustic mismatch for anchor loss control in contour mode resonators}

Cite as: Appl. Phys. Lett. 114, 103502 (2019); https://doi.org/10.1063/1.5086156

Submitted: 18 December 2018 . Accepted: 27 February 2019. Published Online: 13 March 2019

Andrea Lozzi, (D) Annalisa De Pastina, Ernest Ting-Ta Yen, and (i) Luis Guillermo Villanueva
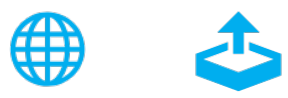

View Online

\section{ARTICLES YOU MAY BE INTERESTED IN}

Effective quality factor tuning mechanisms in micromechanical resonators

Applied Physics Reviews 5, 041307 (2018); https://doi.org/10.1063/1.5027850

Limit cycles and bifurcations in a nonlinear MEMS resonator with a 1:3 internal resonance Applied Physics Letters 114, 103103 (2019); https://doi.org/10.1063/1.5085219

"Möbius" microring resonator

Applied Physics Letters 114, 101106 (2019); https://doi.org/10.1063/1.5082675
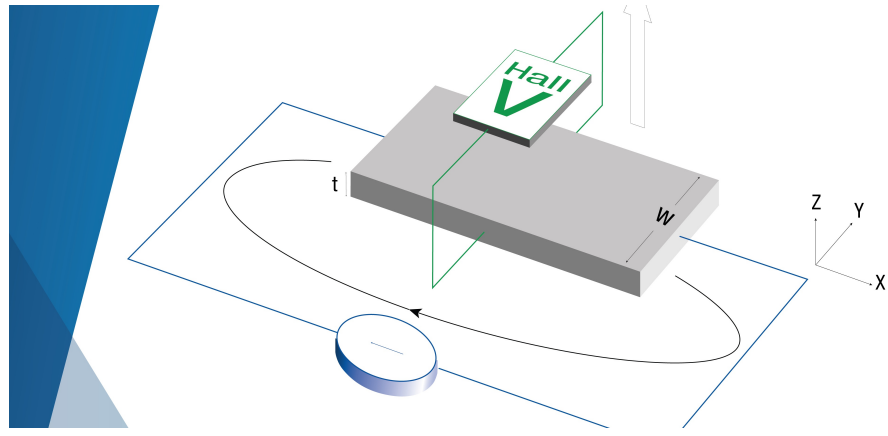

\section{Tips for minimizing} Hall measurement errors

Download the Technical Note 


\title{
Engineered acoustic mismatch for anchor loss control in contour mode resonators
}

\author{
Cite as: Appl. Phys. Lett. 114, 103502 (2019); doi: 10.1063/1.5086156 \\ Submitted: 18 December 2018 - Accepted: 27 February 2019 . \\ Published Online: 13 March 2019
}

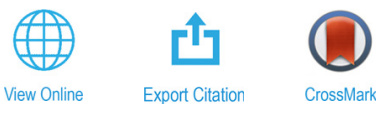

\author{
Andrea Lozzi, ${ }^{1, a)}$ (D) Annalisa De Pastina, ${ }^{7}$ (D) Ernest Ting-Ta Yen, ${ }^{2}$ and Luis Guillermo Villanueva ${ }^{1}$ (iD
}

AFFILIATIONS

${ }^{7}$ Advanced NEMS Lab, École Polytechnique Fédérale de Lausanne (EPFL), Route Cantonale, 1015 Lausanne, VD, Switzerland

${ }^{2}$ Kilby Labs, Texas Instruments Inc., 2900 Semiconductor Dr., Santa Clara, California 95051, USA

a)andrea.lozzi@epfl.ch

\begin{abstract}
Improving the quality factor $(Q)$ of electromechanical resonators is of paramount importance for different applications, ranging from $\mathrm{RF}$ filtering to sensing. In this paper, we present a modified fabrication process flow for contour mode resonators to simultaneously obtain the (i) $Q$ insensitive to the Si undercut geometry and (ii) in-phase reflectors for anchor loss control and $Q$ optimization. To assess the potential of the reflector, we vary its distance from the resonator's anchor. This results in a periodic trend of $Q$ when the distance between the anchor and the reflector changes. Further confirmation of the trend is obtained via a finite element (FE) model. Interestingly, when in the FE model, the step between consecutive reflectors is decreased by a factor of $6 \times$ with respect to the experimental step, we observe a fast modulation of $Q$, superimposed onto that seen experimentally. The origin of this fast modulation is likely the coexistence of waves with different wavelengths traveling through the released region. Our results show that the profile of the region undergoing Si undercutting (released area) can be easily set by design. Furthermore, engineering the introduced acoustic mismatch provides unprecedented control of anchor loss.
\end{abstract}

Published under license by AIP Publishing. https://doi.org/10.1063/1.5086156

Microelectromechanical systems (MEMS) are currently the object of intense research as solutions in timing and filtering systems to replace non-IC compatible blocks. ${ }^{1}$ The major goal is to provide an alternative to quartz and off-chip components and, more importantly, to meet new and more stringent requirements imposed by technological advancements. Size, power consumption, cost, and system integration complexity are becoming limiting factors for many applications, ranging from domestic to medical. Higher performance in those areas will promote a new set of applications, at the moment held back by the lack of the needed hardware (e.g., low or near-zero power wireless radio systems ${ }^{2}$ ) such as the wireless body area network (WBAN) and the internet of things (IoT) in general.

In this context, piezoelectric contour mode resonators (CMRs) are particularly appealing MEMS resonators because of the possibility to set the resonance frequency by layout design. ${ }^{4}$ However, their performance are lower than those of bulk acoustic wave (BAW) resonators, ${ }^{5}$ limiting the competition.

From experimental measurements, it is found that two parameters are generally extracted to assess the performance of a piezoelectric MEMS resonator: the quality factor $Q$ and the electromechanical coupling $k_{t}^{2}$. With the latter being mainly limited by the piezoelectric material, in this case, aluminum nitride (AIN), we herein focus on the former. $Q$ is a direct measure of the energy that is stored in the resonator with respect to the energy that is lost, per cycle of vibration. High $Q$ is desirable both in oscillators, for good close-in phase noise, and in filters, to achieve sharp roll-off and low insertion losses. ${ }^{2,6}$

In AlN CMRs operating below $500 \mathrm{MHz}, Q$ is mainly determined by acoustic energy radiated outward the resonator through the device tethers, referred to as anchor loss. A geometrical optimization approach of the resonator itself has been widely used to minimize anchor loss. Proper design of the resonator anchors, ${ }^{7-9}$ of the resonator shape, ${ }^{10,11}$ and of the inactive region ${ }^{12}$ has proven to influence $Q$. Recently, a study reported that $Q$ is also influenced by the undercut created beyond the tethers because of isotropic Si etching. A 30\% Q variation within micron variation of the undercut is reported. ${ }^{13}$

The difficulties in predicting the Si undercut have often led to the approximation that the reflections of acoustic waves radiated from the anchor into the substrate are negligible. This assumption results in simplified models, ${ }^{7}$ where perfectly absorbing boundaries (PMLs) are used to quantify acoustic damping. ${ }^{8,14}$ However, it is known that the outer end of the anchor acts as a source of circular waves and that these waves propagate until they reach the interface between the released area and the substrate. ${ }^{15}$ At this point, an acoustic mismatch is encountered, creating a reflected wave. Modelling of this 
phenomenon is complex and is strongly dependent on the shape of the Si undercut [Fig. 1(a)], which is difficult, if not impossible, to accurately know a priori when using the most common fabrication process. ${ }^{16}$ Nevertheless, when the acoustic mismatch is introduced by design, an approximate model can be used. Whether it is a high-tolow (etched slots $)^{17}$ or low-to-high $\left(\mathrm{SiO}_{2}\right.$ trenches in this work) acoustic mismatch, transmission line theory can, in principle, be used. This enables the prediction of the behavior of $Q$ as a function of the distance between the source and the reflector, which is set by design and thus is known. In a previous work, we presented a fabrication process flow to attain very stable $Q$ independent of the $\mathrm{Si}$ etching time: using isolation high-aspect ratio trenches filled with $\mathrm{SiO}_{2},{ }^{18}$ we were able to confine and control the region undergoing $\mathrm{Si}$ etching. ${ }^{19}$ In this paper, we take one step forward and show experimentally that engineering of the isolation trench is easily possible via design and allows partial recovery of the lost energy using in-phase reflection.

In Fig. 1, comparison of a fabricated CMR and a fabricated confined CMR (cCMR) is shown, along with their relative process flows. The modified fabrication process allows the designer to choose which region undergoes Si etching (blue areas in Fig. 1). Since the S0 standing Lamb waves in the resonator translate into production of circular waves at the outer end of the anchor, ${ }^{15}$ we design semi-circular reflectors to match the transmitted wavefront [Fig. 1(b)]. We fabricate devices operating at $220 \mathrm{MHz}$ (acoustic wavelength $\lambda_{C M R}=40 \mu \mathrm{m}$ ) varying the radius of the semi-circular reflectors $\left[\mathrm{R}_{\text {rel }}\right.$ in Figs. $1(\mathrm{~b})$ and 2(a)] from $25 \mu \mathrm{m}$ to $49 \mu \mathrm{m}$ in steps of every $3 \mu \mathrm{m}$ to assess its effect on $Q$. We choose such discretization because it is smaller than $\lambda_{C M R} / 10$, enough to show the dependence predicted by transmission line theory, and achievable with our current technology. Additionally, devices with an anchor width [ $W_{a}$ in Fig. 1(a)] ranging from $10 \mu \mathrm{m}$ to $19 \mu \mathrm{m}$ are included in the design to obtain resonators with diverse anchor loss. ${ }^{7,8}$
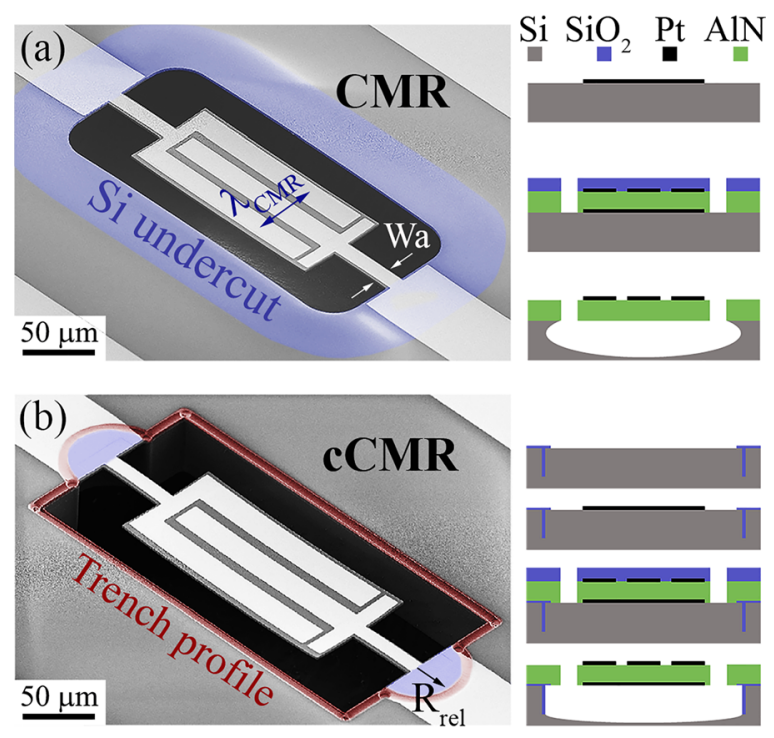

FIG. 1. (a) SEM image of a classic CMR; the undercut created by the isotropic $\mathrm{Si}$ etching to release the structure is highlighted in light blue. (b) SEM picture of a cCMR; the top profile of the high aspect ratio $\mathrm{SiO}_{2}$ trench is highlighted in red, while the semi-circular region that undergoes Si undercutting is highlighted in light blue. On the right, the relative process flows are shown. (a)
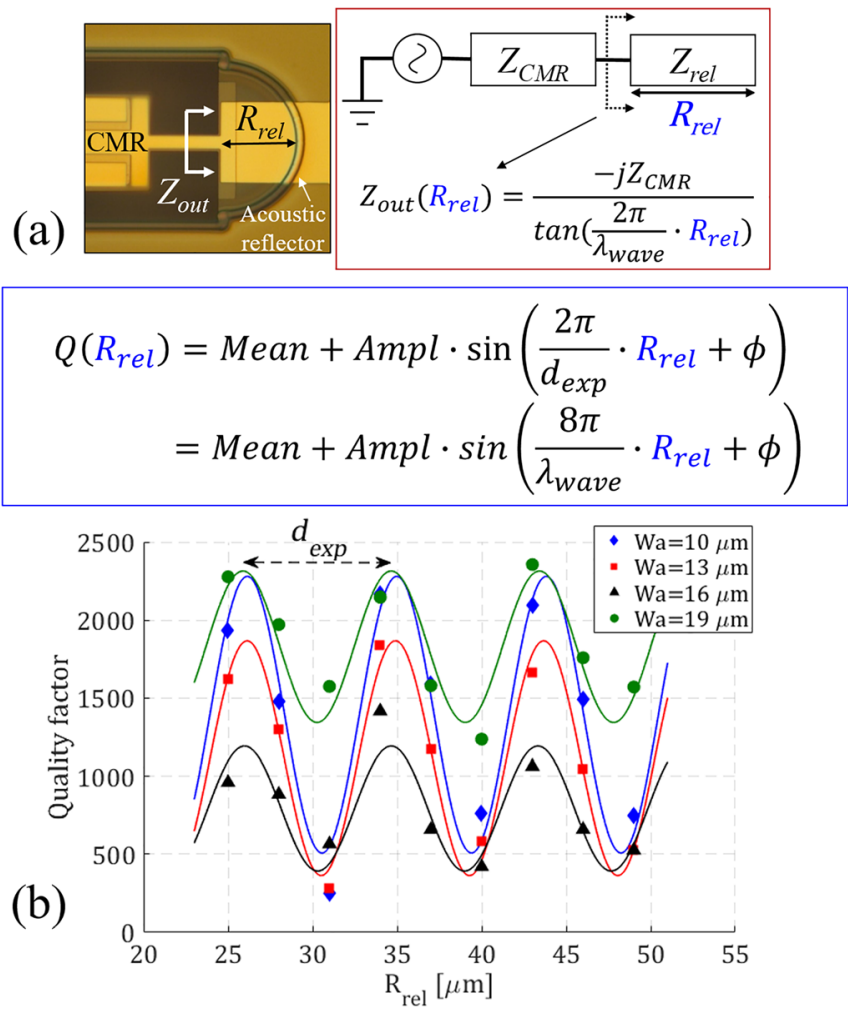

FIG. 2. (a) Optical microscopy image of a cCMR and a transmission line theory model; the impedance seen by the outer end of the anchor is reported in the inset on the top. (b) Experimental data for $W_{a}=10 \mu \mathrm{m}$ (blue diamonds), $W_{a}=13 \mu \mathrm{m}$ (red squares), $W_{a}=16 \mu \mathrm{m}$ (black triangles), and $W_{a}=19 \mu \mathrm{m}$ (green dots) and relative sinusoidal fits (continuous lines); each point is the average $Q$ measured from two identical devices. At the top of the graph, the fitting function for $Q\left(R_{\text {rel }}\right)$ is reported. According to the transmission line theory, the distance between peaks $\left(d_{\text {exp }}\right.$ in the inset) is one quarter of the wavelength.

The fabrication starts with the definition of high-aspect-ratio $\mathrm{SiO}_{2}$ isolation trenches on high resistive $\left(>10000 \Omega \mathrm{cm}^{2}\right)$ wafers. Then, the classic 3-mask process flow is used to fabricate 1-port CMR (Fig. 1). More information about the fabrication of CCMR can be found here. ${ }^{19}$ The purpose of the $\mathrm{SiO}_{2}$ trench is ultimately double: (i) make $Q$ insensitive to Si undercut changes and (ii) create a controlled acoustic mismatch that allows for energy recovery. With the first point being already demonstrated with an improvement of a factor $6 \times$ in $Q$ stability compared to regular $\mathrm{CMR},{ }^{19}$ we herein target the latter point.

Figure 2(b) shows $Q$ as a function of $R_{r e l}$ for four sets of $W_{a}$. It is clear that $Q$ varies periodically as $R_{r e l}$ is swept. In particular, two peaks and two valleys can be clearly identified at $R_{\text {rel }}=34-43 \mu \mathrm{m}$ and $R_{\text {rel }}=31-40 \mu \mathrm{m}$, respectively. Regardless of $W_{a}$, which essentially affects the average value of $Q$, the periodicity of $Q$ is preserved. This proves that the distance (from the outer end of the anchor) at which the reflection occurs has an influence on $Q$ and that the variation is significant, even within only a few micron change in $R_{\text {rel }}$.

With $Q$ being a strong function of $R_{r e l}$, we define the $Q$ recovery $=Q_{\text {delta }} / Q_{\text {average }}$ as the excursion of $Q$ around its mean value when $R_{r e l}$ is varied. This is a measure of how much $Q$ improves from 
the average value, when $R_{r e l}$ is at one of the $Q$ maxima. The values of $Q$ recovery for four different $W_{a}$ values are reported in Table I.

The $Q$ recovery is for all the four cases above $30 \%$. When $W_{a}$ goes from $10 \mu \mathrm{m}\left(\lambda_{C M R} / 4\right)$ to $20 \mu \mathrm{m}\left(\lambda_{C M R} / 2\right)$, the relative excursion of $Q$ around its mean value goes from $\sim 80 \%$ to $\sim 30 \%$. This is due to the fact that values around $W_{a}=\lambda_{C M R} / 2$ minimize the amount of energy radiated outwards from the resonator by the anchor design.? Nevertheless, $31 \%$ Q recovery is observed, confirming that careful anchor design only results in partial anchor loss minimization, which can be further improved with engineering of the released area.

The periodic trend is intuitively explained with in-phase or outof-phase reflections of the transmitted waves. When the reflection is in-phase, $Q$ has a peak because the reflected wave adds up to the standing waves of the resonant mode into the resonator. On the other hand, an out-of-phase reflection creates destructive interference between the reflected wave and the standing waves of the resonant mode, thus lowering $Q$.

We use an open-circuited transmission line to model the reflection as a function of $R_{r e l}$ since the fixed constraint imposed by the substrate can be modeled as an infinite load impedance. ${ }^{20}$ In this way, the distance between the source and the reflector modulates the impedance $\left[Z_{\text {out }}\right.$ in Fig. 2(a)] seen by the outer end of the anchor. When $R_{r e l}$ is at odd multiples of $\lambda_{\text {wave }} / 4$, with $\lambda_{\text {wave }}$ being the wavelength of the wave propagating towards the reflector, the impedance seen is zero, making the outer end of the anchor a virtual stress-free boundary. On the other hand, for even multiples of $\lambda_{\text {wave }} / 4$, the impedance seen is infinite, placing a virtual fixed constraint boundary at the outer end of the anchor. ${ }^{13}$ Any of these two conditions, occurring $\lambda_{\text {wave }} / 4$ apart, results in minimal energy flowing from the CMR into the substrate and thus maximized values of $Q$. As a result, the distance between $Q$ maxima $\left[d_{\text {exp }}\right.$ in Fig. 2(b)] can be used to estimate $\lambda_{\text {wave }}$. The measured $Q$ values as a function of $R_{r e l}$ and $W_{a}$ are thus fitted to a simple sinusoidal function reported in Fig. 2(b). The fit uses a least squares fit algorithm, and all the parameters appearing in the sinusoidal function are left free-running. The extracted $d_{\text {exp }}$ values for different $W_{a}$ designs are reported in Table I. Their value is around $8-9 \mu \mathrm{m}$ for all the cases we study, which is slightly shorter than the designed value of $\lambda_{C M R} / 4=10 \mu \mathrm{m}$, with $\lambda_{C M R}$ being the acoustic wavelength within the resonator's body.

In order to investigate the reasons why $d_{\text {exp }}$ is different from $\lambda_{C M R} / 4$, we decided to look further into the wave propagation in the release area by performing $3 \mathrm{D}$ finite element (FE) modeling.

The FE model, shown in Fig. 3(a), discretizes only one quarter of the device geometry exploiting symmetry boundary conditions in order to minimize the computational cost, yet allowing the definition

TABLE I. $Q$ recovery and extracted distance between peaks for different $W_{a}$ values.

\begin{tabular}{lcccc}
\hline \hline & \multicolumn{4}{c}{ Experimental data } \\
\cline { 2 - 5 } & $Q_{\min }$ & $Q_{\max }$ & $Q_{\text {recovery }}=Q_{\text {delta }} / Q_{\text {average }}[\%]$ & $d_{\text {exp }}(\mu \mathrm{m})$ \\
\hline$W_{a}=10 \mu \mathrm{m}$ & 249 & 2167 & 79 & 8.85 \\
$W_{a}=13 \mu \mathrm{m}$ & 279 & 1835 & 73 & 8.78 \\
$W_{a}=16 \mu \mathrm{m}$ & 419 & 1416 & 54 & 8.68 \\
$W_{a}=19 \mu \mathrm{m}$ & 1236 & 2355 & 31 & 8.78 \\
\hline \hline
\end{tabular}
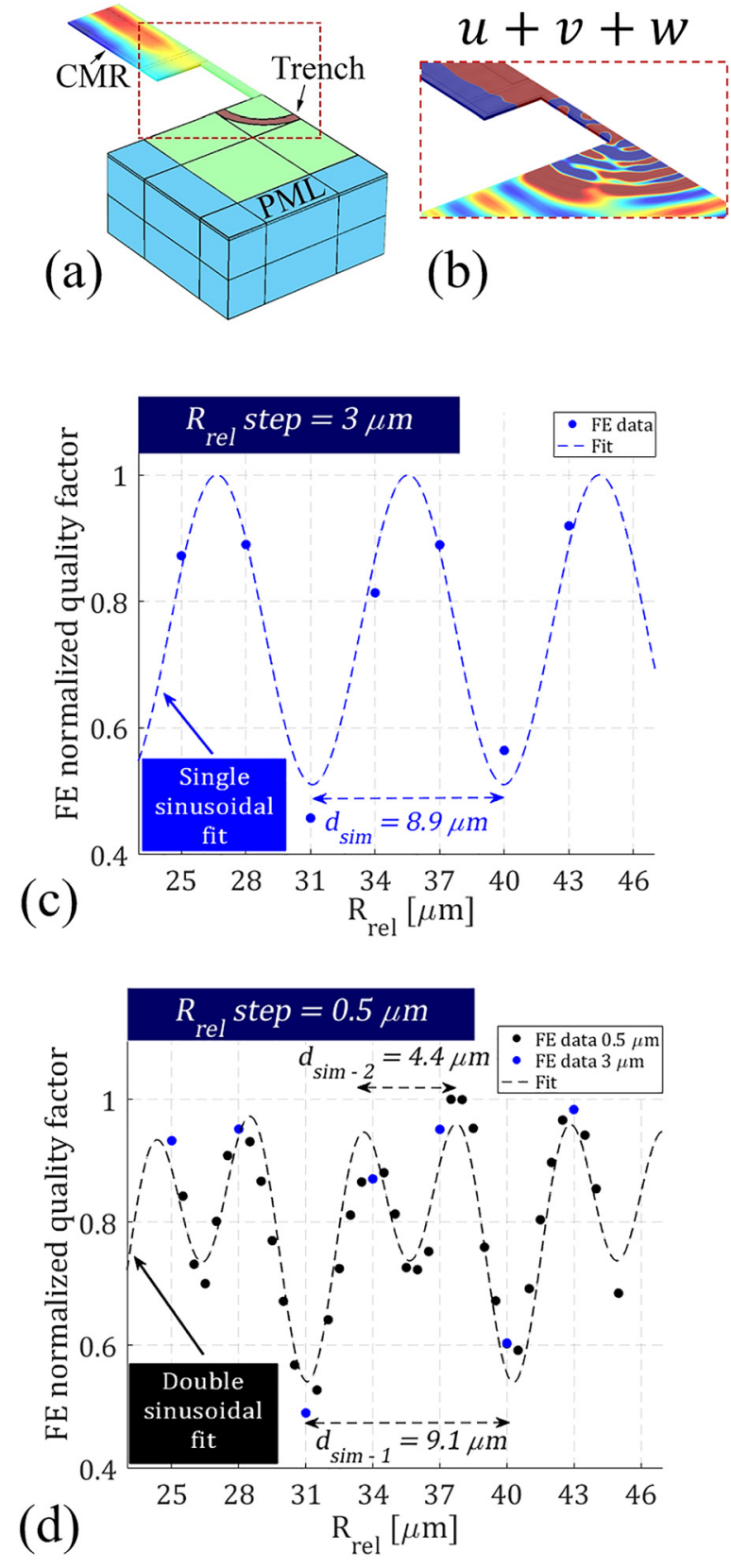

FIG. 3. (a) FE model used to predict the $Q$ trend as a function of $R_{\text {rel. }}$. Only one quarter of the entire geometry is discretized to decrease the computational cost. The mode shape (lateral displacement shown here) can be seen, along with the circular trench and the absorbing layer (PML) used to estimate Q. (b) Zoom-in view of the semi-circular released area; a lateral displacement in the resonator is converted into almost circular waves in the released area. (c) Extracted normalized $Q$ via FE analysis as a function of $R_{\text {rel }}$. To compare with experimental data, numerically obtained $Q$ values starting from $R_{\text {rel }}=25 \mu \mathrm{m}$ for every $3 \mu \mathrm{m}$ are fitted to a single sinusoidal function (blue dashed line). (d) Extracted normalized $Q$ via $F E$ analysis as a function of $R_{\text {rel, }}$ using $0.5 \mu \mathrm{m} R_{\text {rel }}$ step. An additional fast modulation is seen. In this case, the data are fit to a double sinusoidal function (black dash line) confirming the presence of two waves beating. The fast modulation is filtered out by the experimental coarse $R_{\text {rel }}$ step. 
of small features such as the electrodes and the trench. Additionally, a perfectly matched layer (PML) is used to simulate the infinite substrate surrounding the resonator. The PML was dimensioned to half of $\lambda_{C M R} / 2$, after confirming that larger PMLs do not significantly change the value of the extracted $Q .{ }^{8}$

The first attempt is to match the experimental data with the FE model maintaining the $3 \mu \mathrm{m}$ experimental step for $R_{\text {rel }}$. As can be seen in Fig. 3(c), the FE $Q$ values exhibit peaks and valleys at the same $R_{\text {rel }}$ of the experimental data [Fig. 3(c) versus Fig. 2(b)]. Afterwards, to investigate if the mismatch between $d_{\text {exp }}$ and the theoretical value is due to a too coarse $R_{\text {rel }}$ step, we reduce it to $0.5 \mu \mathrm{m}$. This step would be very challenging to replicate experimentally, due to fabrication limitations. The finer step reveals the presence of a fast and a slow modulation in the $Q$ trend [Fig. 3(d)]. In order to extract both, a fit using the sum of two sinusoidal functions is used. Out of the two pulsations obtained via the fit, $d_{\text {sim- } 1}=9.1 \mu \mathrm{m}$, while $d_{\text {sim-2 }}=4.4 \mu \mathrm{m}$. The fast modulation, as expected, is experimentally filtered out when a sampling rate lower than double the periodicity is used. Conversely, the slow modulation is seen as the Nyquist criterion is met (sampling rate $\left.>2 \cdot d_{\text {exp }}\right)$. It is reasonable to think that in the release area region, unlike in the resonator body, the less stringent boundary conditions allow the waves with different wavelengths to coexist at the same frequency. Also, from the data extracted using the FE model and the subsequent fit, the two sinusoidal functions are nearly in phase with each other, meaning that in order to coexist, the two modes spatially share the same nodal point (point of null displacement). For this reason, the fast modulation is a multiple of the slow modulation.

In conclusion, this paper presents a modified process flow that allows fabrication of CMRs with a controlled released area. The goal is double: (i) to stabilize $Q$ that is heavily affected by minor variation of the released area ${ }^{19}$ and (ii) to engineer the released area so as to create in-phase reflectors for anchor loss minimization. A circular released area is designed, and its radius is varied. A periodic trend of $Q$ is observed as the radius is swept. The experimental data are fitted to a transmission line theory model and the extracted distance between $Q$ peaks is slightly lower than the theoretical reference $\lambda_{C M R} / 4$. To investigate the mismatch between the extracted wavelength and the acoustic wavelength set into the resonator by design, a FE model is used. Maintaining the same $R_{r e l}$ step, the simulated $Q$ has the same trend as the experimental data. However, when a finer $R_{r e l}$ step is used, the superimposition of a slow and a fast modulation of $Q$ is revealed. While the slow modulation is caught experimentally, the fast modulation is filtered out because of the low sampling rate. The two modulations are likely due to the presence of waves of diverse nature and with different lambda values coexisting in the release area. Ultimately, this fabrication technique allows the design of CMRs with a stable and reproducible $Q$ across the wafer, independent of CMR geometry and $\mathrm{Si}$ release etching time. This technique, along with design optimization, provides unprecedented control of anchor loss.

The authors would like to acknowledge Professor Romain Fleury for fruitful discussions and the Center for Micronanotechnology (CMi) for their support in the fabrication.

This work was funded by Grant No. PP00P2_170590 of the Swiss SNF and by the CMRs Radios project from Texas Instruments.

\section{REFERENCES}

${ }^{1}$ H. Bhugra and G. Piazza, Piezoelectric MEMS Resonators (Springer, 2017).

${ }^{2}$ C. T.-C. Nguyen, IEEE Commun. Mag. 51(4), 110 (2013).

${ }^{3}$ C. T.-C. Nguyen, IEEE Trans. Ultrason., Ferroelectr., Freq. Control 54(2), 251-270 (2007).

${ }^{4}$ G. Piazza, P. J. Stephanou, and A. P. Pisano, J. Microelectromech. Syst. 15(6), 1406 (2006).

${ }^{5}$ J. Rosenbaum, Bulk Acoustic Wave Theory and Devices (Artech House on Demand, 1988).

${ }^{6}$ D. B. Lesson, Proc. IEEE 54(2), 329 (1966).

${ }^{7}$ J. Segovia-Fernandez, M. Cremonesi, C. Cassella, A. Frangi, and G. Piazza, J. Microelectromech. Syst. 24(2), 265 (2015).

${ }^{8}$ A. Lozzi, L. G. Villanueva, and E. T.-T. Yen, paper presented at the 2016 IEEE International Frequency Control Symposium (IFCS), 2016.

${ }^{9}$ F.-C. Hsu, J.-C. Hsu, T.-C. Huang, C.-H. Wang, and P. Chang, Appl. Phys. Lett. 98(14), 143505 (2011).

${ }^{10}$ C.-M. Lin, Y.-J. Lai, J.-C. Hsu, Y.-Y. Chen, D. G. Senesky, and A. P. Pisano, Appl. Phys. Lett. 99(14), 143501 (2011).

${ }^{11}$ J. Zou, C.-M. Lin, G. Tang, and A. P. Pisano, IEEE Electron Device Lett. 38(12), 1739 (2017).

${ }^{12}$ C. Cassella, N. Singh, B. W. Soon, and P. Gianluca, J. Microelectromech. Syst. 24(5), 1575-1582 (2015).

${ }^{13}$ B. Gibson, K. Qalandar, C. Cassella, G. Piazza, and K. L. Turner, IEEE Trans. Ultrason., Ferroelectr., Freq. Control 64(5), 898-904 (2017).

${ }^{14}$ A. Frangi, M. Cremonesi, A. Jaakkola, and T. Pensala, Sens. Actuator, A 190, 127 (2013).

${ }^{15}$ B. P. Harrington and R. Abdolvand, J. Micromech. Microeng. 21(8), 085021 (2011).

${ }^{16}$ J. Segovia-Fernandez and G. Piazza, J. Microelectromech. Syst. 25(3), 459 (2016).

${ }^{17}$ M. Cremonesi, A. Frangi, C. Cassella, and G. Piazza, Procedia Eng. 87, 468 (2014).

${ }^{18}$ A. De Pastina, D. Maillard, and L. G. Villanueva, Microelectron. Eng. 192, 83 (2018).

${ }^{19}$ A. Lozzi, A. De Pastina, L. G. Villanueva, and E. T.-T. Yen, paper presented at the 2017 IEEE International Ultrasonics Symposium (IUS), 2017.

${ }^{20}$ D. M. Pozar, Microwave Engineering (John Wiley \& Sons, 2009). 